\title{
Efectos de la Fritura al Vacío en los Atributos de Calidad de Arepa con Huevo
}

\author{
José D. Torres*, Diofanor Acevedo y Piedad M. Montero \\ Universidad de Cartagena, Grupo de Investigación Nutrición, Salud y Calidad Alimentaria (NUSCA), \\ Avenida el Consulado, Calle 30 No. 48-152. Cartagena, Bolívar-Colombia. \\ (e-mail: jtorresg3@unicartagena.edu.co) \\ * autor a quien debe ser dirigida la correspondencia
}

Recibido Feb. 11, 2016; Aceptado Abr. 18, 2016; Versión final Jun. 22, 2016, Publicado Feb. 2017

\section{Resumen}

La fritura al vacío permite mejorar la calidad de los alimentos debido a las menores temperaturas y baja exposición del aceite al oxígeno. El objetivo de este estudio fue analizar el efecto del freído al vacío sobre la calidad de arepas con huevo. Se realizaron tres formulaciones con incorporación de pasta de ajonjolí. El freído fue a $120^{\circ} \mathrm{C}, 130^{\circ} \mathrm{C}$ y $140^{\circ} \mathrm{C}, 30 \mathrm{kPa}$ y $5 \mathrm{~min}$. Se utilizó un diseño experimental factorial $3^{2}$. Los contenidos de humedad y luminosidad disminuyeron con el aumento de la temperatura. El aceite aumentó con la adición de pasta. La deseabilidad máxima fue 0.74 a $133.5^{\circ} \mathrm{C}$ y $63.4 \%$ de pasta. Las respuestas fueron: humedad $(30.78 \%)$, aceite $(26.65 \%), \Delta \mathrm{E}(22.74)$, luminosidad (52.41), color (4.24), olor (4.33), sabor (4.64) y dureza (4.45). La fritura al vacío es una tecnología alternativa para obtener arepas con huevo de buena calidad y aceptabilidad.

Palabras clave: arepa con huevo, atributos sensoriales, optimización, pasta de ajonjolí

\section{Effects of Vacuum Frying on the Attributes of Quality of Arepa with Egg}

\begin{abstract}
Vacuum frying improves food quality due to the lower temperature and low oxygen exposure of the frying oil. The aim of this study was to analyze the effect of vacuum frying on the quality of arepas containing egg. Three formulations were made with addition of sesame paste. Frying was at $120^{\circ} \mathrm{C}, 130^{\circ} \mathrm{C}$ and $140{ }^{\circ} \mathrm{C}, 30$ $\mathrm{kPa}$ and $5 \mathrm{~min}$. A factorial experimental design $3^{2}$ was used. Moisture content and brightness decreased with increase in temperature. Oil content increased with the addition of pasta. The maximum desirability was 0.74 at $133.5{ }^{\circ} \mathrm{C}$ and $63.4 \%$ paste. The values of the responses were: moisture $(30.78 \%)$, oil $(26.65 \%)$, $\Delta \mathrm{E}(22,74)$, light $(52,41)$, color $(4,24)$, smell $(4,33)$, flavor $(4.64)$ and hardness $(4,45)$. Vacuum frying is an alternative technology for obtaining arepas with egg with good quality and acceptability.
\end{abstract}

Keywords: arepa with egg, sensory attributes, optimization, sesame paste 


\section{INTRODUCCIÓN}

En la actualidad, el sector de los alimentos fritos ha experimentado un crecimiento significativo, especialmente el consumo de snacks, papas fritas, tortillas de maíz, otros derivados de productos de origen vegetal y los denominados comidas rápidas. Debido a lo anterior, la gran importancia de profundizar en el conocimiento del proceso de freído en los distintos alimentos (Yuksel y Kayacier, 2016). La fritura es uno de los métodos de procesamiento térmico de alimentos más populares e importantes en todo el mundo (Tirado et al., 2012; 2013; Mehrjardi et al., 2012). Puede definirse como un tipo especial de cocción por inmersión en aceite o grasa comestible a una temperatura superior al punto de ebullición del agua (Bouchon, 2009; Tirado et al., 2013; 2015b). Básicamente, la fritura es un proceso de deshidratación como otros (Tirado et al., 2015a; 2016a; 2016b) que origina en los alimentos reacciones de gelatinización de almidones, desnaturalización de proteínas y cambios en las propiedades físicas y organolépticas (Neethu et al., 2016). Además de los cambios muy apreciados por los consumidores, un efecto adicional es la preservación del alimento como resultado de la inactivación de enzimas por calor y la reducción de la actividad de agua (Dueik y Bouchon, 2011). Durante la fritura atmosférica, el aceite se somete a altas temperaturas en presencia de agua y aire, provocando la formación de un gran número compuestos a través de reacciones hidrolíticas, térmica y oxidativa (Idowu y Aworh, 2014).

Una alternativa para mejorar la calidad de los productos durante la fritura por inmersión, es la aplicación de vacío (Shyu et al., 2005; Diamante et al., 2015). En la fritura al vacío, los alimentos se sumergen en aceite en un sistema completamente cerrado, donde la presión se disminuye muy por debajo de los niveles atmosféricos, lo cual posibilita que la temperatura de ebullición del agua y del aceite sea menor, permitiendo retirar la humedad de los alimentos con mayor rapidez (Dueik et al., 2014). Entre los alimentos comúnmente utilizados en la fritura a presión reducida se describen manzana (Shyu y Hwang, 2001), zanahoria (Fan et al., 2005), patata (Garayo y Moreira, 2002), batatas amarillas (Esan et al., 2015), Kiwi dorado (Diamante et al., 2012), patatas azules, mangos y judías verdes (Da Silva y Moreira, 2008), donuts (Tan y Mittal, 2006) y filetes de pescado (Andrés-Bello et al., 2010). En la fritura al vacío, al igual que en la atmosférica, se generan de manera simultánea los procesos de transferencia de calor mediante los mecanismos de conducción-convección y la transferencia de masa, que se manifiesta en la pérdida de agua y absorción de aceite del alimento (Tirado et al., 2012; 2014; 2015b). De acuerdo con Da Silva y Moreira, (2008) la fritura al vacío es una opción viable para producir chips de frutas y hortalizas de alta calidad en un tiempo de procesamiento mucho más corto que la fritura convencional y otras técnicas de procesado térmico. Por otra parte, la ausencia de aire durante la fritura al vacío puede inhibir las reacciones de oxidación de lípidos del aceite y el pardeamiento (Fan et al., 2005; Shyu et al., 2005). Por lo tanto, los alimentos deshidratados producidos por fritura al vacío pueden tener una textura crujiente, un buen color, sabor y adecuada retención de nutrientes (Diamante et al., 2015).

Recientemente, con la alta demanda y consumo de alimentos fritos, la industria ha investigado productos nutritivos para el consumo humano, bajos en grasa y de excelente calidad (Bouchon, 2009; Esan et al., 2015). Los productos fritos son de gran aceptación por parte de los consumidores, debido a sus especiales características sensoriales, derivadas de la presencia de una cantidad de grasa que mejora su textura y les confiere un flavor característico (Mehrjardi et al., 2012). Éste resulta de la formación de compuestos en el medio de fritura y el alimento a través de reacciones como la de Maillard (Su et al., 2016). Recientemente se ha informado que es indispensable la optimización del proceso de fritura, ya que cuando los alimentos ricos en almidón y proteínas se someten a temperaturas por encima de $120{ }^{\circ} \mathrm{C}$, puede haber formación de compuestos potencialmente tóxicos como la acrilamida, furfurales entre otros (Pedreschi et al., 2014).

La arepa con huevo es uno de los alimentos fritos típicos más reconocidos e importantes en la Región Caribe Colombiana. Es elaborada con masas de maíz amarillo a la cual se le adiciona agua y un poco de sal. Actualmente está convertida en una tendencia gastronómica y forma parte de la cultura ancestral típica de Colombia, debido a su gran consumo. El procesamiento de la arepa con huevo es mediante fritura por inmersión, la cual se realiza artesanalmente y no se controla la calidad del producto durante el procesado térmico. Asimismo, no se han reportado estudios que analicen en detalle los parámetros de calidad durante el proceso de fritura al vacío, por lo cual el objetivo de esta investigación fue analizar el efecto del freído al vacío sobre las características de calidad de la arepa con huevo.

\section{MATERIALES Y MÉTODOS}

Se describe la formulación y elaboración de la arepa con huevo, luego se detalla el proceso de fritura por inmersión al vacío y se explica el diseño experimental. Luego se detallan como se obtuvieron los parámetros de calidad de las arepas con huevo freídas al vacío, como sobn el porcentaje de humedad y aceite, los cambios del color y el análisis sensorial. 


\section{Formulación y elaboración de la arepa con huevo}

Las arepas con huevo fueron elaboradas con masas de maíz cariaco amarillo, pre-cocida, la cual fue provista por una empresa comercializadora de masas de maíz, ubicada en la ciudad de Cartagena (BolívarColombia). Con la masa lista, se moldearon las muestras con las dimensiones deseadas, en este caso una placa circular de $10 \mathrm{~cm}$ de diámetro y $2 \mathrm{~cm}$ de espesor. Lo anterior se realizó en un molde apropiado para este fin, el cual poseía una pequeña abertura, para añadir el huevo y el sustituto cárnico. En la Tabla 1 se esquematiza las formulaciones utilizadas para elaborar las arepas con huevo, a la cual se le adicionó como relleno comestible en combinación con el huevo, pasta de ajonjolí que previamente fue elaborada teniendo en cuenta el proceso tecnológico descrito por de Acevedo et al., (2013).

Tabla 1: Formulación de la Arepa con huevo con sustituto cárnico.

\begin{tabular}{|c|c|c|c|c|c|c|}
\hline \multirow{2}{*}{ Ingredientes } & \multicolumn{2}{|c|}{ Formulación 1 } & \multicolumn{2}{c|}{ Formulación 2 } & \multicolumn{2}{c|}{ Formulación 3 } \\
\cline { 2 - 7 } & Cantidad $(\mathrm{g})$ & $(\%)$ & Cantidad $(\mathrm{g})$ & $(\%)$ & Cantidad $(\mathrm{g})$ & $(\%)$ \\
\hline Masa de maíz amarillo & 200 & 68,49 & 200 & 68,49 & 200 & 68,49 \\
\hline Huevos entero crudo & 40 & 13,70 & 40 & 13,70 & 40 & 13,70 \\
\hline Carne molida & $50(100 \%)$ & 17,12 & $25(50 \%)$ & 8,56 & $0(0 \%)$ & 0,00 \\
\hline Pasta de ajonjolí & $0(0 \%)$ & 0,00 & $25(50 \%)$ & 8,56 & $50(100 \%)$ & 17,12 \\
\hline Sal y Pimienta molida & 2 & 0,68 & 2 & 0,68 & 2 & 0,68 \\
\hline Total & 292 & 100 & 292 & 100 & 292 & 100 \\
\hline
\end{tabular}

\section{Proceso de fritura por inmersión al vacío}

Se realizó la fritura al vacío de las muestras de arepas con huevo utilizando aceite de palma en un equipo GASTROVAC ${ }^{\circledR}$ (Cocina Internacional, Barcelona) con medidas de $40 \times 26 \times 46 \mathrm{~cm}$, capacidad máxima de 10,5 L y tensión 220 V (García-Segovia et al., 2016). La presión del proceso fue $30 \mathrm{kPa}$ absolutos y las temperaturas de freído ensayadas fueron de $120^{\circ} \mathrm{C}, 130^{\circ} \mathrm{C}$ y $140{ }^{\circ} \mathrm{C}$ con un tiempo fijo de $300 \mathrm{~s}$. En primer lugar, se calentó el aceite a la temperatura de fritura fijada, se colocaron las muestras de arepas con huevo en el cestillo, se cerró la tapa y se activó la bomba de vacío. Cuando el equipo llegó a la presión deseada, se bajó el cestillo y se sumergió en el aceite caliente. La relación del producto/aceite fue de 1:4 p/v. Una vez cumplido el tiempo de fritura se levantó el cestillo, se dejó durante un minuto la bomba encendida, posteriormente se rompió el vacío y se apagó el equipo retirando las muestras, las cuales fueron escurridas en una canasta de malla metálica a una temperatura ambiente de $25^{\circ} \mathrm{C}$.

\section{Diseño experimental}

En el proceso de freído al vacío de las arepas con huevo elaboradas con sustituto cárnico, se utilizó un diseño experimental en bloque completamente al azar (DBCA) bajo una estructura factorial multinivel de $3^{2}$, dos factores con tres niveles cada uno y una réplica adicional en cada tratamiento, para un total de 18 corridas experimentales. Los factores con sus respectivos niveles bajos, intermedios y altos fueron: $\left(\mathrm{X}_{1}\right)=$ temperatura $\left(120^{\circ} \mathrm{C}, 130{ }^{\circ} \mathrm{C}\right.$ y $\left.140{ }^{\circ} \mathrm{C}\right)$ y $\left(\mathrm{X}_{2}\right)$ = incorporación de pasta de ajonjolí (Formulación $1=0 \%$, Formulación $2=50 \%$ y Formulación $3=100 \%$ ). Las variables respuesta fueron: \% humedad, \% aceite, color instrumental $\left(\mathrm{L}^{*}, \Delta \mathrm{E}\right)$ y las percepciones sensoriales color, olor, sabor y dureza. Estas fueron optimizadas utilizando metodología de superficie de respuesta (MSR) teniendo en cuenta el punto máximo de la deseabilidad, para obtener las mejores arepas con huevo. Las variables independientes se mantuvieron dentro del rango experimental y las respuestas fueron minimizadas (humedad, aceite, $\Delta \mathrm{E}$ ) y maximizadas ( $L^{*}$ y sensoriales). Los modelos de predicción se utilizaron para generar los gráficos de superficie. Los resultados fueron expresados como la media con su respectiva desviación estándar y se compararon utilizando análisis de varianza (ANOVA) y pruebas de comparaciones múltiples a través del test HSD de Tukey con un nivel de significancia del $5 \%$. Se utilizó el programa estadístico Statgrapichs Centurión 16.1 .15 en Windows 8. La matriz de diseño experimental codificado, utilizado en la fritura de arepa con huevo se muestra en la Tabla 2.

Tabla 2: Matriz de diseño experimental codificado, utilizado en la fritura de arepa con huevo

\begin{tabular}{|c|c|c|c|c|c|c|c|c|c|c|c|c|c|c|c|c|c|c|}
\hline Factores/Corridas & 1 & 2 & 3 & 4 & 5 & 6 & 7 & 8 & 9 & 10 & 11 & 12 & 13 & 14 & 15 & 16 & 17 & 18 \\
\hline Pasta de Ajonjolí $(\%)$ & -1 & 0 & 1 & -1 & 0 & 1 & -1 & 0 & 1 & -1 & 0 & 1 & -1 & 0 & 1 & -1 & 0 & 1 \\
\hline Temperatura $\left({ }^{\circ} \mathrm{C}\right)$ & -1 & -1 & -1 & 0 & 0 & 0 & 1 & 1 & 1 & -1 & -1 & -1 & 0 & 0 & 0 & 1 & 1 & 1 \\
\hline
\end{tabular}

El modelo de regresión de segundo orden utilizado para analizar las respuestas del diseño experimental, en función de las variables independientes se esquematiza en la ecuación (1):

$Y=\beta_{0}+\beta_{1} X_{1}+\beta_{2} X_{2}+\beta_{11} X_{1}^{2}+\beta_{22} X_{2}^{2}+\beta_{12} X_{1} X_{2}+\varepsilon$ 
Dónde: $Y=$ variable respuesta; $\beta_{0}=$ intercepto, $X_{1}=$ Temperatura $\left({ }^{\circ} \mathrm{C}\right)$ (Codificados); $\mathrm{X}_{2}=$ pasta de ajonjolí (\%) (Codificados), $\beta_{1}$ y $\beta_{2}=$ Coeficientes para el efecto lineal de $X_{1}$ y $X_{2}$ respectivamente. Además, $\beta_{11}$ y $\beta_{22}$ $=$ Coeficientes para el efecto cuadrático de $X_{1}$ y $X_{2}$ respectivamente, $\beta_{12}=$ Coeficiente para el efecto de la interacción de $\mathrm{X}_{1}$ y $\mathrm{X}_{2}$, y $\varepsilon=$ Error aleatorio.

\section{Porcentaje de humedad y aceite en arepas}

La humedad fue calculada por secado en horno convencional a $105^{\circ} \mathrm{C}$, hasta peso constante de acuerdo a la AOAC 925.10 (AOAC, 2012). Para la determinación del contenido de grasa, se empleó el método Soxhlet, por extracción con éter de petróleo de acuerdo a lo establecido en la AOAC 920.85 (AOAC, 2012). Todas las mediciones fueron realizadas por triplicado y expresadas como el promedio en base seca.

\section{Cambios del Color}

El color de las arepas con huevo, se midió en un colorímetro de reflectancia de laboratorio CR-5 (tristimolo Konica Minolta Sensing), con iluminante D65 y un ángulo $10^{\circ}$. Los parámetros se evaluaron siguiendo la metodología del sistema $C I E L^{*} a^{*} b$, en cuanto a la luminosidad $L^{*}$, que indica claro cuando es próximo a $100^{\circ}$ y oscuro cuando tiende a $0^{\circ}$; cromaticidad $a^{*}$, rojo con valores positivo y verde con negativos y cromaticidad $\mathrm{b}^{*}$, amarillo cuando son positivos y azul cuando son negativos. Así mismo se calculó el cambio general del color $(\Delta \mathrm{E})$ respecto al producto sin procesar (estándar), utilizando la ecuación (2):

$\Delta E=\sqrt{\left(L^{*}-L\right)^{2}+\left(a^{*}-a\right)^{2}+\left(b^{*}-b\right)^{2}}$

Dónde: $\mathrm{L}^{*}=$ tratamiento, $\mathrm{L}=$ estándar, $\mathrm{a}^{*}=$ tratamiento, $\mathrm{a}=$ estándar, $\mathrm{b}^{*}=$ tratamiento, $\mathrm{b}=$ estándar. Todos los ensayos se realizaron por triplicado y los datos se expresaron como el promedio con su respectiva desviación estándar.

\section{Análisis sensorial}

En la cuantificación del nivel de agrado o desagrado de los atributos de: color, olor, sabor y dureza. Se utilizó una escala hedónica estructurada de cinco puntos, las categorías fueron desde "me gusta mucho" (calificación 5) hasta "me disgusta mucho" (calificación 1). Las muestras se presentaron codificadas en orden aleatorio y se utilizó un panel entrenado de 30 catadores, a los cuales se les proporcionó muestras enteras de arepas con huevo freídas al vacío, teniendo en cuenta los tratamientos del diseño experimental. Los datos se recogieron en una planilla y se procesaron mediante análisis de varianza y el test HSD Tukey con un nivel de significancia del $5 \%$.

\section{RESULTADOS Y DISCUSIONES}

En la Tabla 3, se esquematiza el ANOVA multifactorial de las variables respuestas respecto a los factores temperatura de freído al vacío, pasta de ajonjolí y sus respectivas interacciones cuadráticas. Así mismo se muestran los coeficientes determinación $\left(R^{2}\right)$ obtenidos.

Tabla 3: ANOVA de respuestas de arepa con huevo freídas al vacío $\left(p<0,05^{\star} ; p<0,01^{\star *} ; p<0,001^{* \star *}\right)$

\begin{tabular}{|c|c|c|c|c|c|c|c|c|}
\hline Parámetros & $\%$ Humedad & $\%$ Aceite & $\mathrm{L}^{*}$ & $\Delta \mathrm{E}$ & Color & Olor & Sabor & Dureza \\
\hline $\mathrm{X}_{1}$ : Temperatura $\left({ }^{\circ} \mathrm{C}\right)$ & $0,000^{\star \star \star}$ & $0,000^{\star \star \star}$ & $0,000^{\star \star *}$ & $0,000^{\star \star \star}$ & $0,000^{\star \star \star}$ & $0,003^{* *}$ & $0,000^{\star \star \star}$ & $0,001^{\star *}$ \\
\hline $\mathrm{X}_{2}$ : Pasta de ajonjolí & $0,032^{*}$ & $0,015^{\star}$ & $0,000^{\star * \star}$ & $0,009^{*}$ & $0,000^{\star \star *}$ & $0,000^{\star \star \star}$ & $0,000^{\star \star \star}$ & $0,002^{* *}$ \\
\hline$X_{1}^{2}$ & 0,869 & 0,048 & $0,000^{\star * *}$ & $0,038^{*}$ & $0,007^{* *}$ & $0,043^{*}$ & 0,263 & $0,000^{\star *}$ \\
\hline$X_{1} X_{2}$ & 0,969 & $0,008^{*}$ & 0,065 & 0,644 & $0,004^{* \star}$ & $0,003^{* *}$ & 0,134 & 0,322 \\
\hline$X_{2}^{2}$ & 0,463 & 0,097 & 0,098 & 0,376 & $0,001^{* *}$ & 0,571 & $0,000^{\star \star \star}$ & $0,000^{\star \star}$ \\
\hline$R^{2}$ & 0,909 & 0,975 & 0,986 & 0,888 & 0,985 & 0,919 & 0,981 & 0,819 \\
\hline
\end{tabular}

En la pérdida de humedad se encontró que tanto la temperatura como el porcentaje de ajonjolí, fueron estadísticamente significativos $(p<0,05)$ presentando más influencia la temperatura. En el contenido de aceite el efecto lineal de la temperatura y la interacción fueron altamente significativos $(p<0,01)$. Así mismo se observó significancia del factor $\mathrm{X}_{2}$. Indicando que la adición de la pasta de ajonjolí dentro del producto afectó significativamente el contenido final de aceite. Encontrándose un incremento en la absorción de aceite, cuando se aumentó con la cantidad de pasta de ajonjolí. Para la luminosidad y el cambio de color, la temperatura fue el factor que más predominó. De igual manera el factor pasta de ajonjolí fue altamente significativo en las variables respuestas del color, encontrándose que a mayor contenido el producto quedó más oscuro, observándose un comportamiento similar con el aumento de la temperatura. Lo cual posiblemente se debió al desarrollo y formación de componentes derivados de la reacción de Maillard, entre los aminoácidos libres de la zeína de la masa de maíz en este caso lisina, con los azucares reductores de la 
pasta de ajonjolí, la cual contenía un porcentaje de sacarosa que probablemente se caramelizó por acción de las temperaturas y dividió en sus monosacáridos glucosa y fructosa, los cuales presentan un grupo carbonilo reactivo libre en su estructura. Los resultados de los cambios de color para las arepas con huevo freídas al vacío, coinciden con lo reportado por otros autores en productos vegetales como Garayo y Moreira, (2002) en chips de papas y Esan et al., (2015) en batatas amarillas, quienes a su vez indicaron que el desarrollo de color durante el freído al vacío, sólo comienza cuando en el producto se ha producido un secado adecuado. Así mismo Dueik et al., (2014) en matrices con alto contenido de carbohidratos indicaron que los factores tiempo y temperatura del proceso de fritura al vacío, influyen significativamente en los cambios de color, los cuales son definidos por las reacciones de pardeamiento no enzimático en la superficie. De manera similar Troncoso et al., (2009) en trozos de papa y Yuniarti et al., (2012) en chips de piña, encontraron que el desarrollo del color depende del contenido de azúcares reductores y de las condiciones de temperatura. Reportaron además que los productos fueron menos oscuros cuando se procesaron con fritura al vacío.

Según Dueik y Bouchon (2011) la fritura al vacío previene la coloración excesiva producida por quemaduras u oscurecimiento de los snacks de diversas frutas y vegetales; debido a que las reacciones de Maillard, son menores a temperaturas y presiones bajas, lo cual a su vez evita la formación de compuestos tóxicos y potencialmente cancerígenos como acrilamidas, furfurales, uretanos, nitrosaminas. Igualmente, Song et al., (2007) mostraron que la fritura al vacío ayudó a conservar los atributos de calidad y por consiguiente aumentar la vida anaquel de chips de patata. Por su parte Shyu et al., (2005) observaron que el cambio de color de chips de zanahoria se atribuía a la inestabilidad de los carotenoides a partir de $100 \stackrel{\circ}{\circ}$. En general la temperatura y porcentaje ajonjolí, fueron altamente significativos en todos los parámetros sensoriales. Los panelistas indicaron una muy buena aceptación a medida que aumentó la sustitución pasta de ajonjolí; posiblemente este componente, en combinación con el huevo al interior de las muestras influyeron en el desarrollo de aromas que agradaron a los catadores al momento de degustar las arepas.

\section{Optimización de las características de calidad de las arepas con huevo}

En la Tabla 4, se esquematizan los coeficientes de regresión obtenidos de los polinomios ajustados a los datos experimentales; estos describen el comportamiento de las superficies de respuestas que se esquematizan en las Figura 1 (a) (b) (c) (d) (e) (f) (g) (h). Para la humedad, luminosidad y cambio de color se observa un comportamiento lineal, disminuyendo significativamente con el incremento de la temperatura, y el efecto cuadrático de este factor. En cuanto al contenido de aceite y las percepciones de color, sabor y dureza, el factor pasta de ajonjolí tuvo una gran influencia, observándose presencia de curvatura.

Tabla 4: Coeficientes de regresión del modelo estimados para cada variable respuesta

\begin{tabular}{|l|l|l|l|l|l|l|l|l|}
\hline Parámetros & $\%$ Humedad & $\%$ Aceite & $\mathrm{L}^{*}$ & $\Delta \mathrm{E}$ & Color & Olor & Sabor & Dureza \\
\hline Intercepto $\left(\beta_{0}\right)$ & 186,222 & 161,952 & 922,382 & 708,347 & $-25,873$ & 24,995 & 1,773 & 1,773 \\
\hline Temperatura $\left(\beta_{1}\right)$ & $-1,135$ & $-3,024$ & $-11,746$ & $-9,745$ & 0,407 & $-0,323$ & 0,413 & 0,337 \\
\hline Ajonjolí/Carne $\left(\beta_{2}\right)$ & $-0,053$ & 0,256 & $-0,044$ & 0,042 & 0,044 & $-0,031$ & 0,567 & 0,349 \\
\hline Temperatura*Temperatura $\left(\beta_{11}\right)$ & - & 0,015 & 0,039 & 0,034 & $-0,061$ & 0,061 & - & 0,567 \\
\hline Ajonjolí/Carne*Ajonjolí/Carne $\left(\beta_{22}\right)$ & - & $-0,003$ & - & - & $-0,011$ & - & $-0,343$ & 0,446 \\
\hline Temperatura* Ajonjolí/Carne $\left(\beta_{12}\right)$ & - & - & - & - & $-0,023$ & 0,052 & - & - \\
\hline $\mathrm{R}^{2}$ (Ajustada) & 0,884 & 0,963 & 0,965 & 0,859 & 0,974 & 0,873 & 0,975 & 0,783 \\
\hline
\end{tabular}

El coeficiente de determinación $\left(R^{2}\right)$ ajustado para la humedad, fue mayor a 0,80; lo cual indica un buen ajuste de los datos experimentales, y que este modelo fue apropiado para describir el comportamiento lineal de la pérdida de agua al interior de las arepas con huevo durante la fritura al vacío. Así mismo el estadístico $\left(R^{2}\right)$ para el aceite fue mayor al 0,95 . Por su parte el comportamiento de las respuestas de color $L^{*} y \Delta E$, se pueden describir aceptablemente mediante estas ecuaciones, siendo los coeficientes de correlación mayores de 0,95 y 0,85 respectivamente. Las respuestas sensoriales color, olor, sabor y dureza, mostraron coeficientes de determinación ajustados mayores a 0,75; lo que indica que los datos obtenidos del panel sensorial, se ajustaron satisfactoriamente a los modelos cuadráticos utilizados. En estudios de optimización similares Fan et al., (2005) en chips de zanahoria e Idowu y Aworh (2014) en muestras de kokoro un producto típico de Nigeria elaborado con masas de maíz, demostraron que los modelos de regresión se ajustaron adecuadamente a los datos experimentales durante la optimización.

En la presente investigación cuando el proceso de fritura al vacío de las arepas con huevo se realizó por encima de $130^{\circ} \mathrm{C}$, las muestras presentaron mayor pérdida de humedad y a mayor porcentaje ajonjolí el producto presentó más contenido de aceite superficial. La variación de la humedadpueden ser explicada por el concepto de fuerza térmica equivalente durante el freído al vacío (Dueik y Bouchon, 2011; Oginni et al., 2015), ya que la disminución de la presión del sistema ocasiona que la temperatura de ebullición del agua sea menor y por tanto las temperaturas de fritura sean más bajas. Lo cual ocasiona que la humedad del 
alimento rápidamente sea transformada en vapor, que escapa por los poros del producto a una velocidad que aumenta con el delta de temperatura entre el alimento y el medio de procesamiento. Así mismo diversos factores como la porosidad de la matriz alimentaria y el encogimiento (Dueik et al., 2014) pudieron influir en la velocidad de pérdida de humedad durante la fritura al vacío de las arepas con huevo. Estos resultados fueron similares a los encontrados por Yagua et al., (2011) en chips de papas y Diamante et al., (2012) en rodajas de kiwi dorado, quienes reportaron que el contenido de humedad debe ser controlado para asegurar una buena formación de la corteza en la periferia del alimento durante la deshidratación en aceite, y que no se vea afectado la crujencia a medida que se aumentan la temperatura y el tiempo de fritura. Así mismo Oginni et al., (2015) en snacks a base de yuca, reportaron que cuando los productos se retiraron de la freidora, el vapor se condensó en el interior de los poros y la diferencia de presión entre el tejido circundante y el poro hizo que el aceite adherido a la superficie fuera absorbido, lo cual puede ser una posible explicación de la absorción de aceite en las arepas con huevo en la presente investigación.

Por su parte Garayo y Moreira (2002) y Shyu et al., (2005) señalaron que la absorción de aceite estuvo relacionada significativamente con el contenido de humedad de chips de papas y rodajas de zanahoria. Sin embargo, Zhang et al., (2016) quienes estudiaron el proceso de fritura de papas reportaron que el contenido inicial de humedad no tuvo ningún efecto significativo en la absorción de aceite. Mientras que Gazmuri y Bouchon (2009) indicaron que la absorción de aceite no estuvo claramente relacionada con la cantidad de humedad perdida, sino más bien a la microestructura del producto. Lo cual podría explicar la relación perdida de humedad y absorción de aceite encontrado en la presente investigación para arepas con huevo. La formación de la costra en las superficies de las arepas con huevo, se atribuye a las reacciones de gelatinización del almidón de la masa de maíz por acción de las altas temperatura, así mismo a la desnaturalización de la proteína específicamente la zeína que es predominante en el maíz (Idowu y Aworh, 2014). Para las arepas con huevo se encontró además que la corteza superficial se formó rápidamente a medida que aumentó la temperatura, y dicha costra actuó como una barrera a la absorción de aceite; a su vez esta costra permitió mantener un porcentaje de humedad en el interior del alimento, la cual posibilitó un centro totalmente cocido, que fue propicio en la aceptación por parte del panel sensorial. Por otro lado, el aumento de la temperatura durante la fritura, originó cambios en las estructuras de las proteínas del huevo, dicha desnaturalización puede explicarse por el cambio en la conformación de las cadenas de aminoácidos que conforman dichas proteínas, las cuales pasaron de su estado nativo con estructura terciaria y/o cuaternaria, a un estado lineal o primario (Sobukola et al., 2013). Esto llevó posiblemente a que las estructuras se agruparan a través de la formación de puentes de disulfuro, haciendo que la yema y la clara cambiaran de líquidas a sólidas, durante la fritura. En esta investigación el calor alteró las propiedades físicas de la proteína del huevo, pero no cambió los valores nutritivos. Después de cada fritura las temperaturas internas del producto fueron medidas y se encontró que en ningún caso sobrepasaron los 70 ${ }^{\circ} \mathrm{C}$, temperatura a la cual no se alteran significativamente los nutrientes como vitaminas, minerales y proteínas en el alimento.

En un estudio similares de fritura al vacío Sobukola et al., (2013) y Yuksel, y Kayacier (2016) señalaron que la fritura al vacío permite obtener productos deshidratados con buena textura y menores contenidos de humedad y grasa en comparación con la fritura a presión atmosférica, y siendo los cambios producidos en los snacks base de trigo influenciados significativamente por la temperatura $(p<0,05)$. Por su parte Villamizar et al., (2011) reportaron que el freído al vacío fue influenciado significativamente por la temperatura, y dicho proceso mejoró el olor y sabor en muestras de pasa bocas de mango y posibilitó obtener un producto con menor contenido de aceite en comparación con muestras similares freídas a presión atmosférica. Tan y Mittal (2006) procesaron mediante freído al vacío donuts y encontraron que los atributos sensoriales de color, olor, sabor y dureza finales fueron dependientes de los cambios de temperatura del proceso y el producto final presentó un contenido de aceite más alto en comparación con la fritura atmosférica. Da Silva y Moreira (2008) observaron que chips de mango, judías verdes, papas azules y patatas dulces freídas en condiciones atmosféricas fueron de menor calidad que los mismos productos fritos al vacío, aunque las características de textura de los productos fritos no se vieron afectados por los métodos utilizados. También mostraron que el contenido de aceite de los chips de batata y judías verdes fue $24 \%$ y $16 \%$ menor en comparación con los mismos productos freídos convencionalmente. Las batatas azules fritas al vacío y los chips de mango presentaron un $6 \%$ y $5 \%$ más de aceite, que las muestras fritas tradicionalmente.

En el proceso de optimización numérica de múltiples respuestas durante la fritura al vacío de la arepa con huevo Figura 1 (i), se obtuvieron las mejores condiciones de procesamiento a una temperatura de $133,53{ }^{\circ} \mathrm{C}$ y un porcentaje de pasta de ajonjolí del $63,43 \%$ con una deseabilidad máxima $(0,74)$ donde los valores óptimos en las respuestas minimizadas fueron: humedad $(30,78 \%)$, aceite $(26,65 \%), \Delta \mathrm{E}(22,74)$. Mientras que las maximizadas: Iuminosidad $(52,41)$ y percepciones sensoriales color $(4,24)$, olor $(4,33)$, sabor $(4,64)$ y dureza $(4,45)$; lo cual en general indica que el producto elaborado y freído al vacío fue del agrado a los panelistas. 


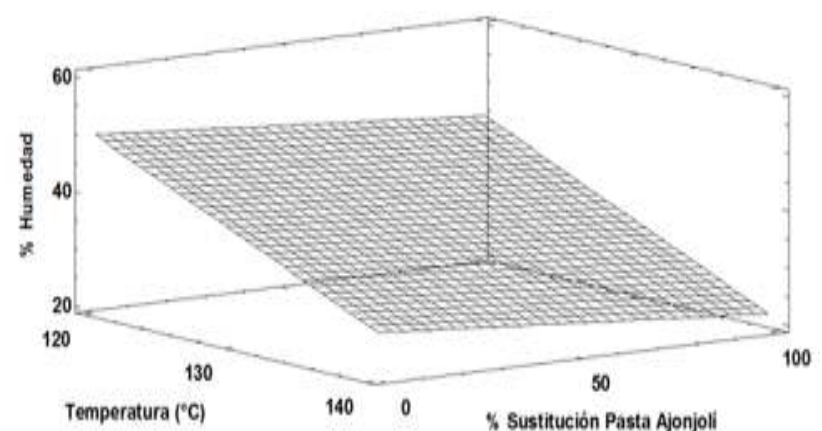

(a) \% Humedad

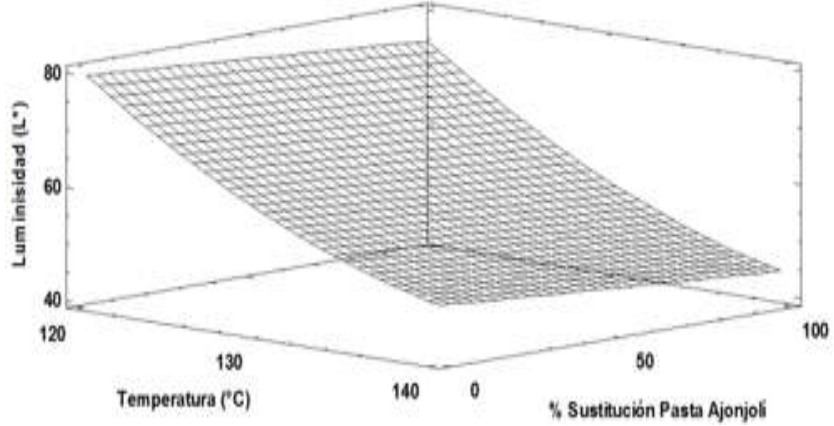

(c) Luminosidad

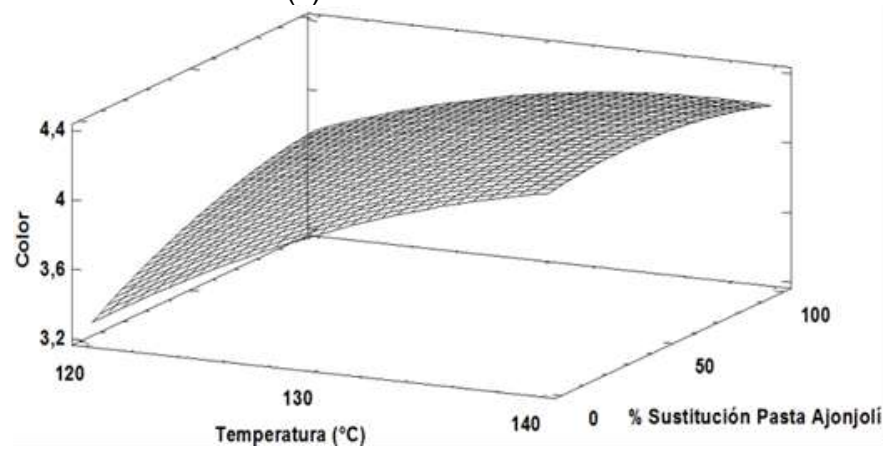

(e) Color

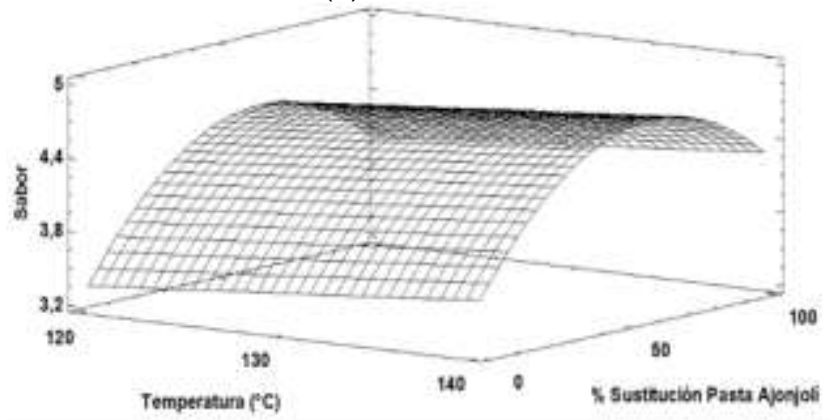

(g) Sabor

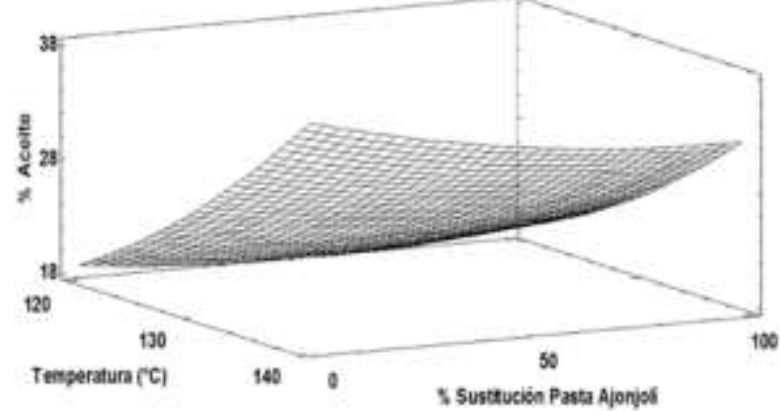

(b) $\%$ Aceite

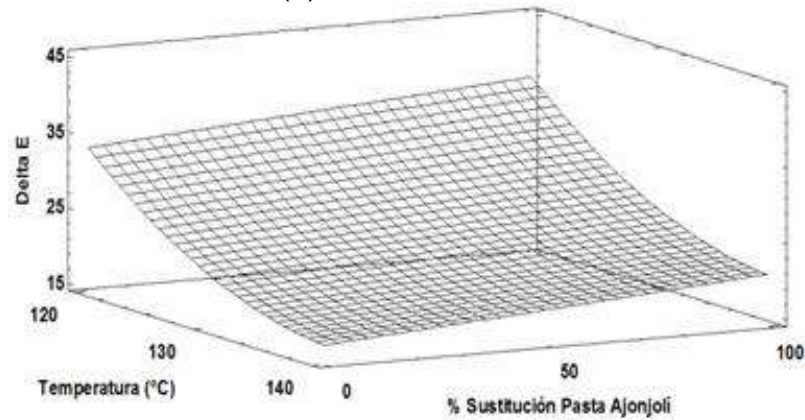

(d) Cambio de color $(\Delta E)$
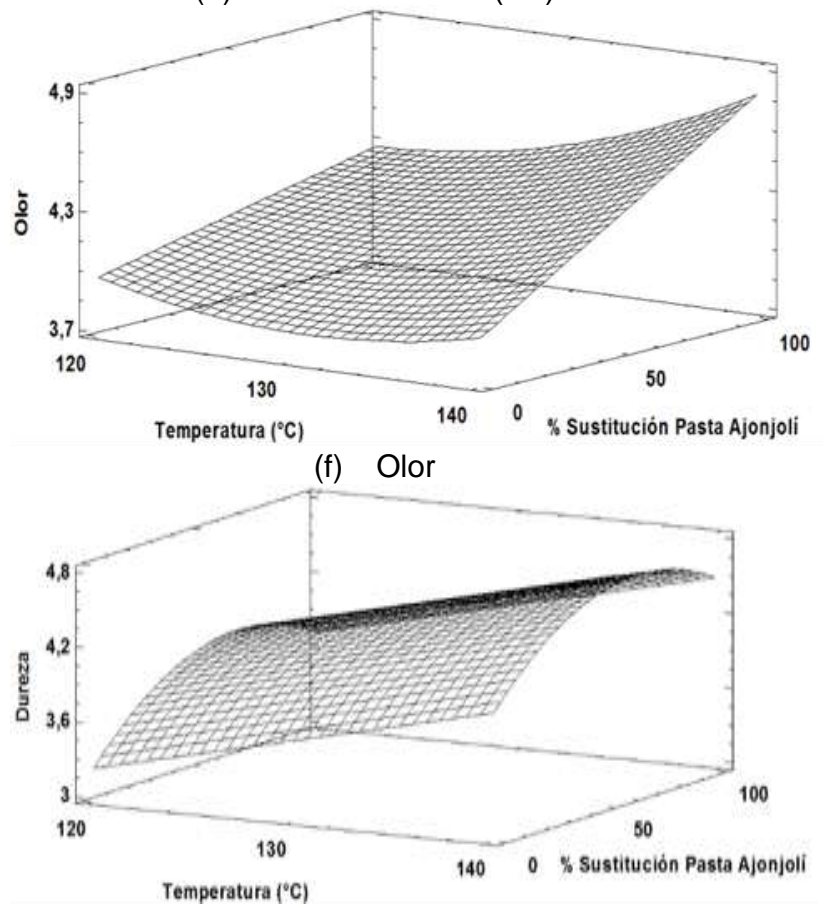

(h) Dureza sensorial

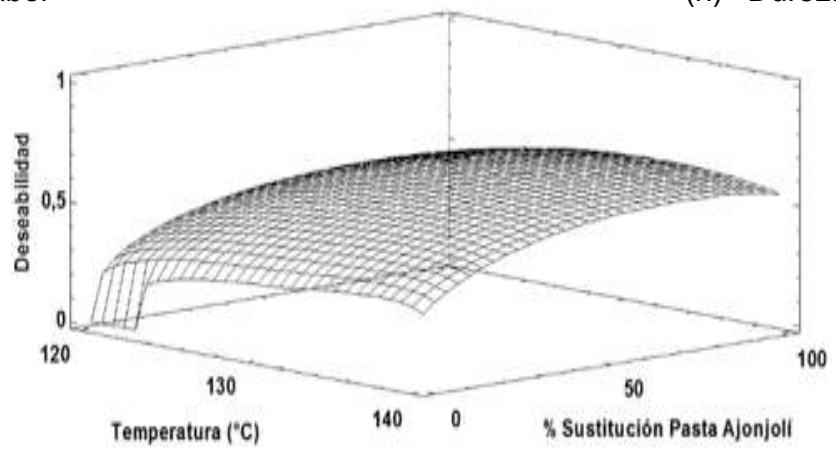

(i) Deseabilidad

Fig. 1: Superficies de respuestas estimadas para los parámetros sensoriales de las arepas con huevo freídas al vacío: (a) Humedad; (b) Aceite; (c) Luminosidad; (d) Cambio de color instrumental; (e) Color sensorial; (f) Sabor; (g) Dureza;

(h) Deseabilidad. 
Estos resultados de optimización de las arepas con huevo, fueron similares a los reportados en la fritura al vacío de galletas de arroz, realizado por Maneerote et al., (2009) quienes encontraron condiciones óptimas minimizando el contenido de aceite a $220^{\circ} \mathrm{C}$ durante 60 segundos. Por su parte Omidiran et al., (2016) en la optimización de los atributos de calidad de los aperitivos fritos con harina de trigo y harina de yuca, encontraron que la deseabilidad máxima fue de 0,77 con una temperatura de fritura de $140{ }^{\circ} \mathrm{C}$, y tiempo de 4 minutos; y los análisis sensoriales mostraron que la muestra optimizada fue aceptaba en términos de la textura, aroma, sabor y la apariencia. Igualmente, Akinpelu et al., (2014) basados en el concepto de la máxima deseabilidad encontraron condiciones óptimas de fritura al vacío a $133^{\circ} \mathrm{C}$, presión de $9,91 \mathrm{~cm}$ de $\mathrm{Hg}$ y tiempo de 6 minutos. Mientras que Shyu y Hwang (2001) en chips de manzanas reportaron rangos óptimos de temperatura entre $100-110{ }^{\circ} \mathrm{C}$, con tiempos de 20 a 25 minutos. Así mismo Diamante et al., (2012) en la optimización de la fritura al vacío de trozos de kiwi dorado, encontraron que condiciones óptimas a temperaturas entre 72,1 y $76,3^{\circ} \mathrm{C}$ con tiempos de 35 a 65 minutos; y Esan et al., (2015) en batatas amarillas reportaron Las condiciones óptimas para fritura al vacío a una presión de vacío de 10,12 $\mathrm{cm}$ de $\mathrm{Hg}, 108^{\circ} \mathrm{C}$ y 9 minutos; donde la deseabilidad máxima fue de 0,61. Además, estos autores indicaron que la fritura al vacío es una alternativa para producir chips patatas de buena calidad nutricional.

\section{CONCLUSIONES}

La humedad de las arepas con huevo, disminuyó significativamente con el aumento de la temperatura de fritura y del factor pasta de ajonjolí. El contenido de aceite en las muestras fue influenciado por la temperatura y se acrecentó significativamente con la adición de pasta de ajonjolí. El incremento de ambos factores ocasionó un descenso significativo en la luminosidad $\left(\mathrm{L}^{*}\right)$ y la variación del color $(\Delta \mathrm{E})$ del producto. La temperatura y porcentaje ajonjolí, influyeron positiva y significativamente en todos los parámetros sensoriales. La deseabilidad máxima fue 0,74 ; a una temperatura de $133,53{ }^{\circ} \mathrm{C}$ y un porcentaje de pasta de ajonjolí de $63,43 \%$. El producto elaborado mediante fritura al vacío fue ampliamente aceptado por los panelistas, especialmente en las percepciones de olor y sabor. Los coeficientes de determinación $\mathrm{R}^{2}$ ajustados, estuvieron por encima de 0,7 en todas las variables respuesta. La fritura al vacío es una alternativa para procesar y obtener arepas con huevo con adecuadas características de calidad y aceptabilidad.

\section{AGRADECIMIENTOS}

Los autores agradecen a la Universidad de Cartagena y a COLCIENCIAS por el apoyo y financiación al Grupo de Investigación Nutrición, Salud y Calidad Alimentaria (NUSCA) en el proyecto 110766441899.

\section{REFERENCIAS}

Acevedo, D., Y. Marrugo. y P. Montero, Evaluación de las propiedades reológicas de pastas de ajonjolí artesanal y tecnificada, Revista UDCA Actualidad \& Divulgación Científica, 16(1), 245-251 (2013)

Akinpelu, O.R. y otros seis autores, Optimization of processing conditions for vacuum frying of high quality fried plantain chips using response surface methodology (RSM), Food Science and Biotechnology, 23(4), 1121-1128 (2014)

Andrés-Bello, A., P. García-Segovia y J. Martínez-Monzó, Vacuum frying process of gilthead sea bream (Sparus aurata) fillets, Innovative Food Science \& Emerging Technologies, 11(4), 630-636 (2010)

AOAC., Association of Official Analytical Chemists. Official Methods of Analysis. 19th ed. AOAC. Arlington. VA, USA. (2012)

Bouchon, P., Understanding oil absorption during deep-fat frying. Advances in food and nutrition research, 57, 209-234 (2009)

Da Silva, P.F. y R.G. Moreira, Vacuum frying of high-quality fruit and vegetable-based snacks, LWT-Food Science and Technology, 41(10), 1758-1767 (2008)

Diamante, L. M. y otros tres autores, Vacuum frying foods: products, process and optimization, International Food Research Journal, 22(1), 15-22 (2015)

Diamante, L.M., G.P. Savage y L. Vanhanen, Optimisation of vacuum frying of gold kiwifruit slices: application of response surface methodology, International Journal of Food Science \& Technology, 47(3), 518-524 (2012) 
Dueik, V. y P. Bouchon, Development of healthy low fat snacks: Understanding the mechanisms of quality changes during atmospheric and vacuum frying, Food Reviews International, 27(4), 408-432 (2011)

Dueik, V., O. Sobukola y P. Bouchon, Development of low-fat gluten and starch fried matrices with high fiber content, LWT-Food Science and Technology, 59(1), 6-11 (2014)

Esan, T.A. y otros cuatro autores, Process optimization by response surface methodology and quality attributes of vacuum fried yellow-fleshed sweetpotato (Ipomoea batatas L.) chips, Food and Bioproducts Processing, 95, 27-37 (2015)

Fan, L.P. y otros cuatro autores, The optimization of vacuum frying to dehydrate carrot chips, International Journal of Food Science \& Technology, 40(9), 911-919 (2005)

Garayo, J. y R. Moreira, Vacuum Frying of Potato Chips, Journal of Food Engineering, 55(2), 181-191. (2002)

García-Segovia, P. y tres autores, Effects of processing conditions on the quality of vacuum fried cassava chips (Manihot esculenta Crantz), LWT-Food Science and Technology, 69, 515-521 (2016)

Gazmuri, A.M. y P. Bouchon, Analysis of wheat gluten and starch matrices during deep-fat frying, Food Chemistry, 115(3), 999-1005 (2009)

Idowu, A.O. y C.O. Aworh, Optimization of some processing conditions for Kokoro production using Response Surface Methodology, Agricultural Engineering International: CIGR Journal, 16(2), 187-195 (2014)

Idowu, A.O. y C.O. Aworh, Optimization of some processing conditions for Kokoro production using Response Surface Methodology, Agricultural Engineering International: CIGR Journal, 16(2), 187-195 (2014)

Maneerote, J., A. Noomhorm y P.S. Takhar, Optimization of processing conditions to reduce oil uptake and enhance physico-chemical properties of deep fried rice crackers, LWT-Food Science and Technology, 42(4), 805-812 (2009)

Mehrjardi, P., B. Tarzi y A. Bassiri, Developing Vacuum Fried Pumpkin (Cucurbita Moschata Dutch) snack, World Applied Sciences Journal, 18(2), 214-220 (2012)

Neethu, K.C. y otros cuatro autores, Prediction of convective heat transfer coefficient during deep-fat frying of pantoa using neurocomputing approaches, Innovative Food Science \& Emerging Technologies, 34, 275$284(2016)$

Oginni, O.C. y otros cuatro autores, Effect of starch gelatinization and vacuum frying conditions on structure development and associated quality attributes of cassava-gluten based snack, Food Structure, 3, 12-20 (2015)

Omidiran, A.T. y otros seis autores, Optimization of some processing parameters and quality attributes of fried snacks from blends of wheat flour and brewers' spent cassava flour, Food science \& nutrition, 4(1), 8088 (2016)

Pedreschi, F., M.S. Mariotti y K. Granby, Current issues in dietary acrylamide: formation, mitigation and risk assessment, Journal of the Science of Food and Agriculture, 94(1), 9-20 (2014)

Shyu, S.L. y L.C. Hwang, Effects of processing conditions on the quality of vacuum fried apple chips, Food Research International, 34(2), 133-142 (2001)

Shyu, S.L.; L.B. Hau y L.S. Hwan, Effects of processing conditions on the quality of vacuum-fried carrot chips, Journal of the Science of Food and Agriculture, 85(11), 1903-1908 (2005)

Sobukola, O.P., V. Dueik y P. Bouchon, Understanding the effect of vacuum level in structure development and oil absorption in vacuum-fried wheat starch and gluten-based snacks, Food and Bioprocess Technology, 6(8), 2010-2017 (2013)

Song, X.J., M. Zhang y A.S. Mujumdar, Effect of vacuum-microwave predrying on quality of vacuum-fried potato chips, Drying Technology, 25(12), 2021-2026, (2007) 
Su, Y., M. Zhang y W. Zhang, Effect of low temperature on the microwave-assisted vacuum frying of potato chips, Drying Technology, 34(2), 227-234 (2016)

Tan, K.J. y G.S. Mittal, Physicochemical properties changes of donuts during vacuum frying, International Journal of Food Properties, 9(1), 85-98 (2006)

Tirado, D.F., D. Acevedo y L.E. Guzmán, Coeficientes convectivos de transferencia de calor durante el freído de láminas de tilapia "Oreochromis niloticus", Información Tecnológica, 24(6), 41-46 (2013)

Tirado, D.F., D. Acevedo y L.E. Guzmán, Freído por inmersión de los alimentos, Revista Reciteia, 12(1), 69$82(2012)$

Tirado, D.F., D. Acevedo y P. Montero, Cinética de transferencia de masa durante salado de arenca (Triportheus magdalenae), Interciencia, 40(2), 127-132 (2015a)

Tirado, D.F., D. Acevedo y P. Montero, Transferencia de Calor y Materia durante el Proceso de Freído de Alimentos: Tilapia (Oreochromis niloticus) y Fruta de Pan (Artocarpus communis), Información Tecnológica, 26(1), 85-94 (2015b)

Tirado, D.F., D. Acevedo y P. Montero, Secado de arenca (Triportheus magdalenae): Influencia del salado en pila seca y húmeda, Revista de Biotecnología en el Sector Agropecuario y Agroindustrial, 14(2), 57-64 (2016a)

Tirado, D.F., D. Acevedo y P. Montero, Secado de rodajas de fruto del árbol del pan mediante la técnica de Ventana Refractiva $\AA$, Tecno Lógicas, 19(36), 103-111 (2016b)

Tirado, D.F., D. Acevedo y P. Puello, Determinación Computacional del Coeficiente de Transferencia de Calor y Propiedades Termofísicas de Alimentos, Información Tecnológica, 25(3), 53-58 (2014)

Troncoso, E., F. Pedreschi y R. Zúñiga, Comparative study of physical and sensory properties of pre-treated potato slices during vacuum and atmospheric frying, LWT - Food Science and Technology, 42(2), 187-195 (2009)

Villamizar, R.H., M.C. Quinceno y G.A. Giraldo, Comparación de la fritura al vacío y atmosférica en la obtención de pasabocas de mango (Manguifera indica L.), Temas Agrarios,16(1), 64-74 (2011)

Yagua, C. y R. Moreira, Physical and Thermal properties of potato chips during vacuum frying, Journal of Food Engineering, 104(2), 272-283 (2011)

Yuksel, F., y A. Kayacier, Utilization of stale bread in fried wheat chips: Response surface methodology study for the characterization of textural, morphologic, sensory, some physicochemical and chemical properties of wheat chips, LWT-Food Science and Technology, 67, 89-98 (2016)

Yuniarti, Y., y otros tres autores, Determination of frying temperatura and vacuum pressure to produce pineapple chips using simple vacuum frier, Journal Technology Pertanian, 4(3), 129-132 (2012)

Zhang, T. y otros tres autores, Effects of Initial Moisture Content on the Oil Absorption Behavior of Potato Chips During Frying Process, Food and Bioprocess Technology, 9(2), 331-340 (2016) 